\title{
BMJ Open Assessment of patient and provider attitudes towards therapeutic drug monitoring to improve medication adherence in low-income patients with hypertension: a qualitative study
}

Kevin B Schesing (D) , ${ }^{1}$ Ricardo Chia, ${ }^{2}$ Bryan Elwood, ${ }^{3}$ Ethan A Halm, ${ }^{3}$ Simon J Craddock Lee, ${ }^{4}$ Hamza Lodhi, ${ }^{2}$ Bryan Wu, ${ }^{1}$ Shishir Sharma, ${ }^{5}$ Scott A Smith, ${ }^{6}$ Robin B Jarrett, ${ }^{7}$ Sandeep R Das, ${ }^{5,8}$ Wanpen Vongpatanasin ${ }^{2,5}$

To cite: Schesing KB, Chia R, Elwood B, et al. Assessment of patient and provider attitudes towards therapeutic drug monitoring to improve medication adherence in low-income patients with hypertension: a qualitative study. BMJ Open 2020;10:e039940. doi:10.1136/ bmjopen-2020-039940

- Prepublication history for this paper is available online To view these files, please visit the journal online (http://dx.doi. org/10.1136/bmjopen-2020039940).

Received 30 April 2020 Revised 30 0ctober 2020 Accepted 09 November 2020

D) Check for updates

(C) Author(s) (or their employer(s)) 2020. Re-use permitted under CC BY-NC. No commercial re-use. See rights and permissions. Published by BMJ.

For numbered affiliations see end of article.

Correspondence to Dr Wanpen Vongpatanasin; wanpen.vongpatanasin@ utsouthwestern.edu

\section{ABSTRACT}

Objectives Previous studies have implicated therapeutic drug monitoring (TDM), by measuring serum or urine drug levels, as a highly reliable technique for detecting medication non-adherence but the attitudes of patients and physicians toward TDM have not been evaluated previously. Accordingly, we solicited input from patients with uncontrolled hypertension and their physicians about their views on TDM.

Design Prospective analysis of responses to a set of questions during semistructured interviews.

Setting Outpatient clinics in an integrated health system which provides care for a low-income, uninsured population.

Participants Patients with uncontrolled hypertension with either systolic blood pressure of at least $130 \mathrm{~mm} \mathrm{Hg}$ or diastolic blood pressure of at least $80 \mathrm{~mm} \mathrm{Hg}$ despite antihypertensive drugs and providers in the general cardiology and internal medicine clinics.

Primary and secondary outcome measures Attitudes towards TDM and the potential impact on physicianpatient relationship.

Results We interviewed 11 patients and 10 providers and discussed the findings with 13 community advisory panel (CAP) members. Of the patients interviewed, $91 \%$ (10 of 11) and all 10 providers thought TDM was a good idea and should be used regularly to better understand the reasons for poorly controlled hypertension. However, $63 \%$ (7 of 11) of patients and $20 \%$ of providers expressed reservations that TDM could negatively impact the physician-patient relationship. Despite some concerns, the majority of patients, providers and CAP members believed that if test results are communicated without blaming patients, the potential benefits of TDM in identifying suboptimal adherence and eliciting barriers to adherence outweighed the risks.

Conclusion The idea of TDM is well accepted by patients and their providers. TDM information if delivered in a nonjudgmental manner, to encourage an honest conversation between patients and physicians, has the potential to reduce patient-physician communication obstacles and to identify barriers to adherence which, when overcome, can improve health outcomes.
Strengths and limitations of this study

- The strength of the study is the first study that explored the attitudes of patients and clinicians regarding the use of therapeutic drug monitoring in clinical practice, which is important since the concept of drug testing could be interpreted as a lack of trust.

- Another strength of the study is additional input from an independent group of community advisory panel.

- The limitation of the study is the small sample size and the study setting is limited to indigent care population, which may not be applicable to all healthcare systems.

\section{INTRODUCTION}

Non-adherence to antihypertensive medications is a major contributor to cardiovascular disease events globally. ${ }^{1}$ At least half of patients with hypertension are non-adherent to treatment within 1 year of treatment initiation. $^{2-5}$ In the USA, it was estimated that approximately 16.3 million $(31 \%)$ of insured US adults with hypertension are nonadherent to their prescribed antihypertensive therapy. ${ }^{6}$ Given the enormous burden of the problem, pragmatic strategies that improve detection and medication adherence are urgently needed to optimise cardiovascular outcomes.

An increasing number of studies by our group and others have indicated that therapeutic drug monitoring (TDM), biochemical monitoring of drug levels in the serum or urine, constitutes a highly reliable technique for detecting medication non-adherence in patients with hypertension not be captured by conventional methods such as patient selfreport or pharmacy refill data. ${ }^{2-47}$ In addition, 
biochemical screening is widely available for clinical use and covered by most health insurance carrier in the USA and many countries worldwide. ${ }^{2}{ }^{8}$ Several small observational studies have indicated that when non-adherent patients were given TDM-guided feedback regarding specific undetectable drug levels, blood pressure (BP) improved substantially at subsequent visits despite no significant change in their prescribed antihypertensive regimen and no intensive counselling beyond that typically provided at a regular clinic visit. ${ }^{27}$

While TDM appears promising, the attitudes of patients and clinicians regarding the use of TDM in clinical practice have not been assessed, especially since the concept of drug testing could be interpreted as a lack of trust. Given the higher rates of medical mistrust among low income and minority populations, negative attitudes towards TDM might be a major barrier to implementation among the very high-risk groups with uncontrolled hypertension this approach is designed to help. ${ }^{9}$ Therefore, we conducted in-depth semistructured interviews of low-income patients and their providers, using a combination of scripted and open-ended follow-up questions, to understand: (1) their knowledge, attitudes, beliefs and concerns about using a blood test to monitor medication adherence and (2) how best to introduce and use TDM in a respectful, patient-centred way.

\section{METHODS}

This study was approved by the University of Texas Southwestern Medical Center Institutional Review Board. After an informed consent was obtained, we conducted semistructured interviews with 11 patients who have uncontrolled hypertension, defined as systolic blood pressure (SBP) of at least $130 \mathrm{~mm} \mathrm{Hg}$ or diastolic blood pressure (DBP) of at least $80 \mathrm{~mm} \mathrm{Hg}$ despite treatment with $\geq 2$ antihypertensive drugs, from the general internal medicine and cardiology clinics of Parkland Health \& Hospital Systems (PHHS). Exclusion criteria include: (1) presence of hypertensive emergency (BP $>180 / 110 \mathrm{~mm} \mathrm{Hg}$ plus one of the following features: acute coronary syndrome, acute stroke, hypertensive encephalopathy, aortic dissection or acute kidney injury), (2) history of active substance abuse such as alcohol, cocaine or narcotics, (3) uncontrolled psychiatric disorder such as schizophrenia or major depression based on diagnosis entered in electronic medical record, (4) pregnancy, (5) homelessness, (6) stage $\mathrm{V}$ Chronic kidney disease (CKD) or end stage renal disease (glomerular filtration rate $<15 \mathrm{~mL} / \mathrm{min} / 1.73 \mathrm{~m}^{2}$ ), (7) self-report of non-adherence or unwillingness to follow medication regimen prescribed by the primary care physicians for any reasons, (8) presence of white coat hypertension (defined as normal 24 hours ambulatory SBP or home BP of $<130$ and diastolic BP of $<80 \mathrm{~mm}$ $\mathrm{Hg}$ and clinic SBP of at least $140 \mathrm{~mm} \mathrm{Hg}$ or diastolic BP of at least $90 \mathrm{~mm} \mathrm{Hg}$ and (9) inability to read or write English. PHHS is an integrated health system which serves as the safety-net hospital for a large, low income, uninsured population in the Dallas County, Texas, USA. We also interviewed 10 providers (physicians, mid-level practitioners and clinical pharmacists) who work with low-income patients with poorly controlled hypertension in the Cardiology and General Internal Medicine clinics. Patients and clinicians received a US\$20 gift card for their time and participation.

The interviews were designed to assess: (1) how TDM could be used in a way that is acceptable to patients that enhances both the patient-provider relationship and improves patient health outcomes, (2) potential causes of non-adherence, (3) patient's reluctance to reveal nonadherence to their provider, (4) how TDM could contradict patient self-report about adherence and (5) how this contradiction could potentially affect patient-provider trust and communication. The interview guide comprised nine questions that explored respondents' personal and/ or professional experiences with treatment of hypertension, with the blood test for therapeutic monitoring, and to explore perspectives on how the test may best be used in practice. The interview scripts were tested and refined after initial practice sessions. Follow-up questions were added to elaborate answers and pursue complementary lines of inquiry if the initial response to the main questions was unclear. A patient/provider interview script is shown in table 1 .

Interviews were digitally recorded, transcribed and analysed under the guidance of the Patient-Centered Outcomes Research (PCOR) Center's Qualitative Methods Core. ${ }^{10}$ Transcripts were coded by three independent coders using a two-step deductive/inductive approach to thematic content analysis. ${ }^{11}$ After reading all the transcripts, we applied initial open codes derived from the interview guide topics. Line-by-line coding led to understanding and development of thematic categories of codes, using highlighting and margin coding of recurring and relevant themes. Each reviewer coded the transcripts independently then met to discuss their individual findings with the larger team and resolve coding discrepancies by consensus. This iterative process enabled the team to refine and revise codes accordingly. ${ }^{12}$ The codes identified fell under the categories of adherence barriers, adherence facilitators, barriers to disclosure, monitoring rationale and intervention implications. We also performed a descriptive statistical analysis to assess the proportion of patients or providers who responded to each question in different ways. We ceased recruitment on reaching thematic saturation.

The rationale and proposed methods for the study were also presented to the community advisory panel (CAP) to seek additional input. The CAP programme is curated by the UT Southwestern Center for PCOR to engage community perspectives on the relevance, design and implications of research that has the potential to improve patient-centred care. The members comprise a diverse racial and ethnic group of lay individuals with experience with the PHHS health system or community-based 


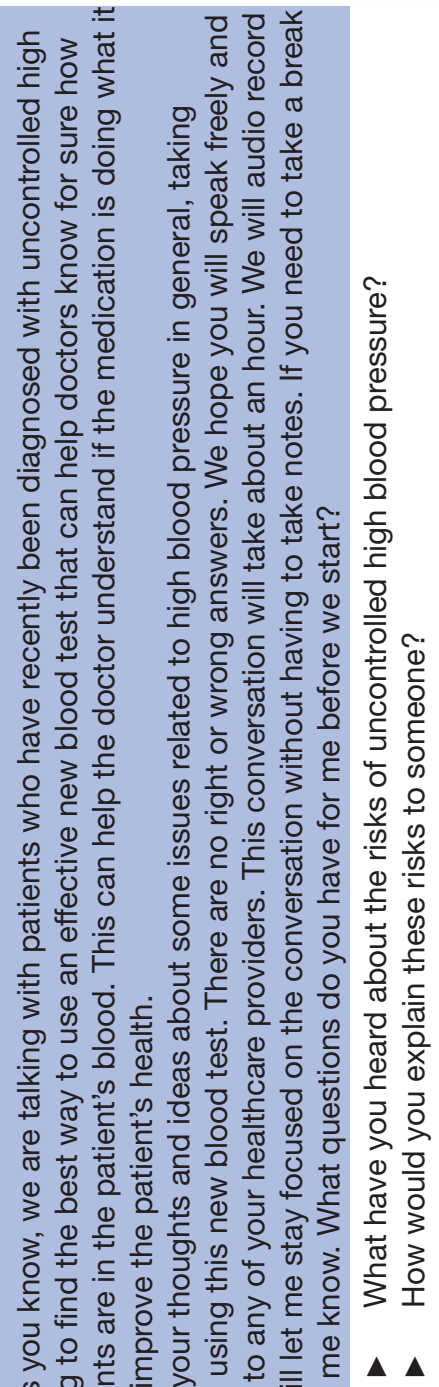
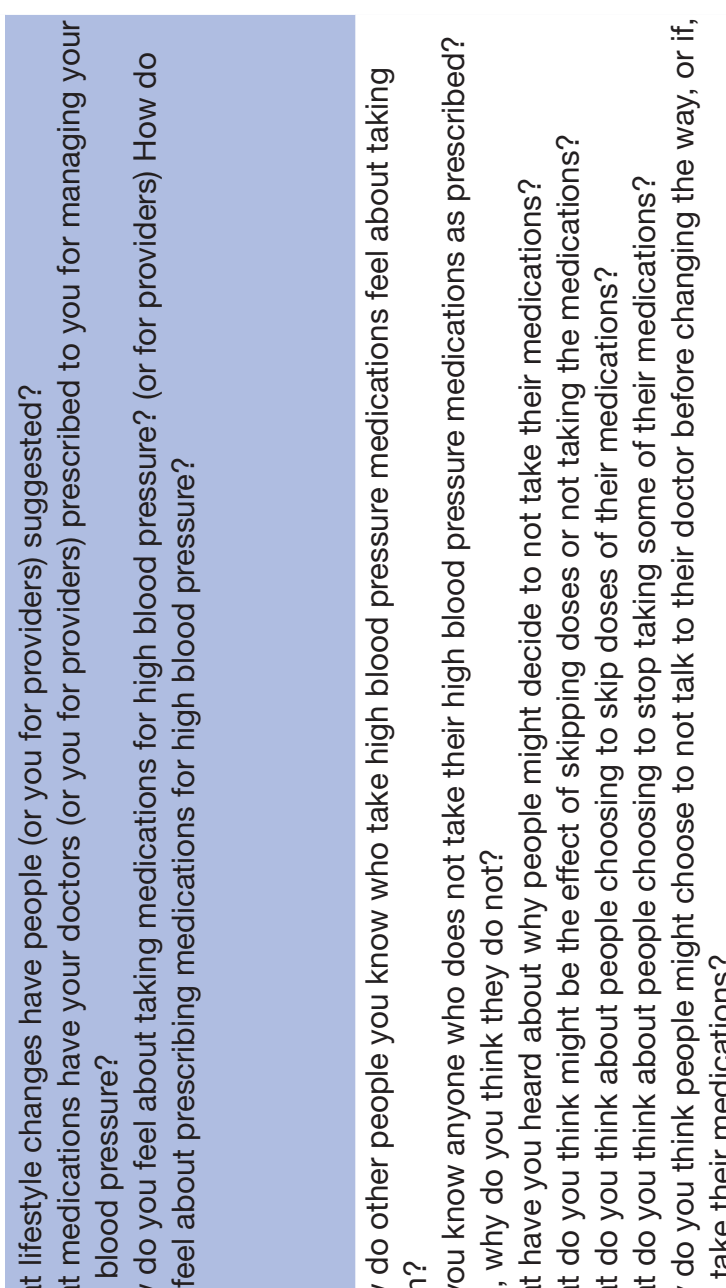

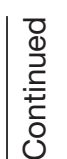

足. 일.

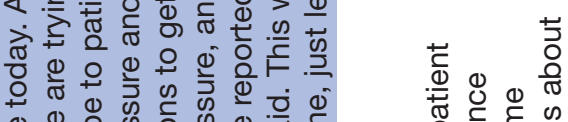

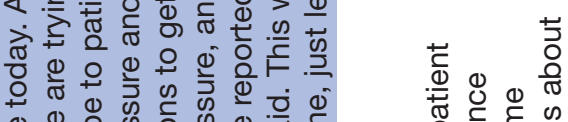

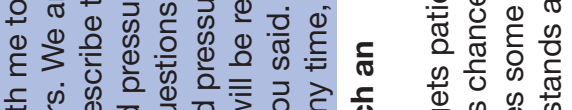
造市

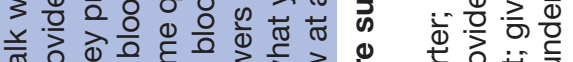

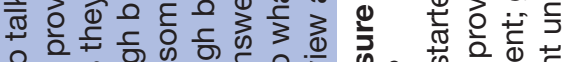
은

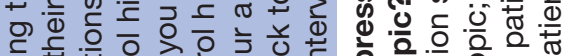

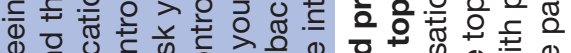
帝 ๖

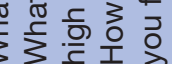

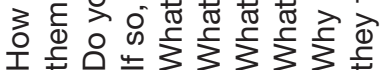
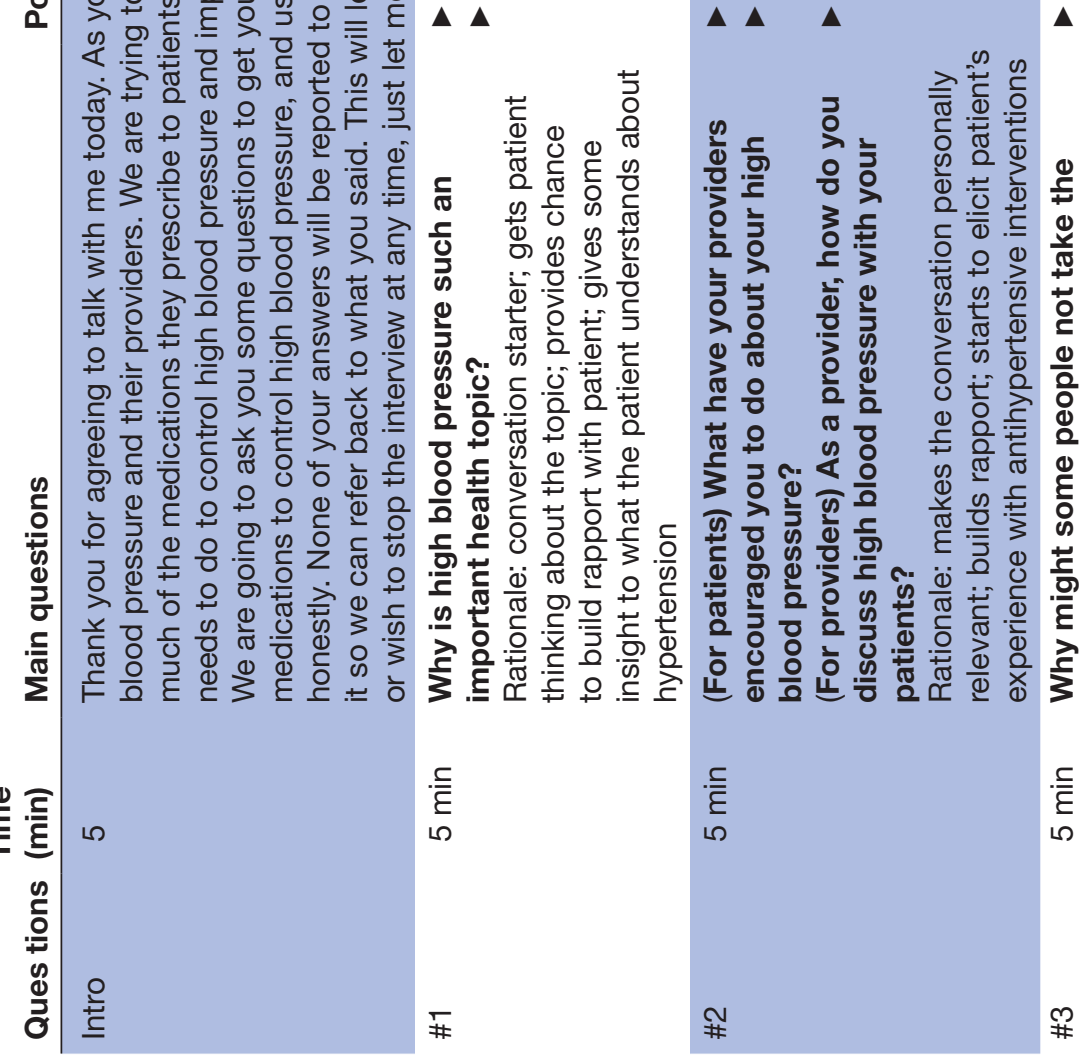


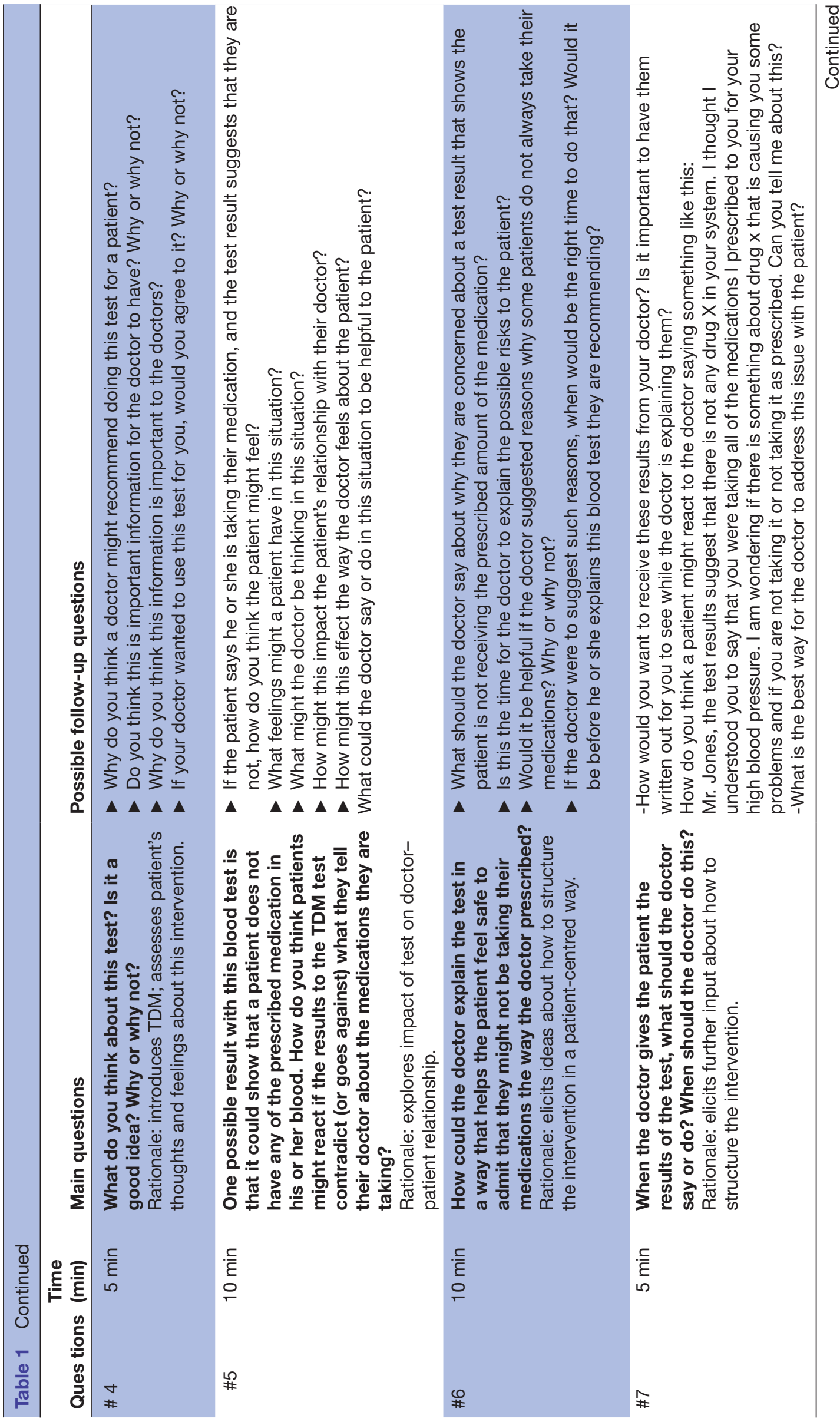




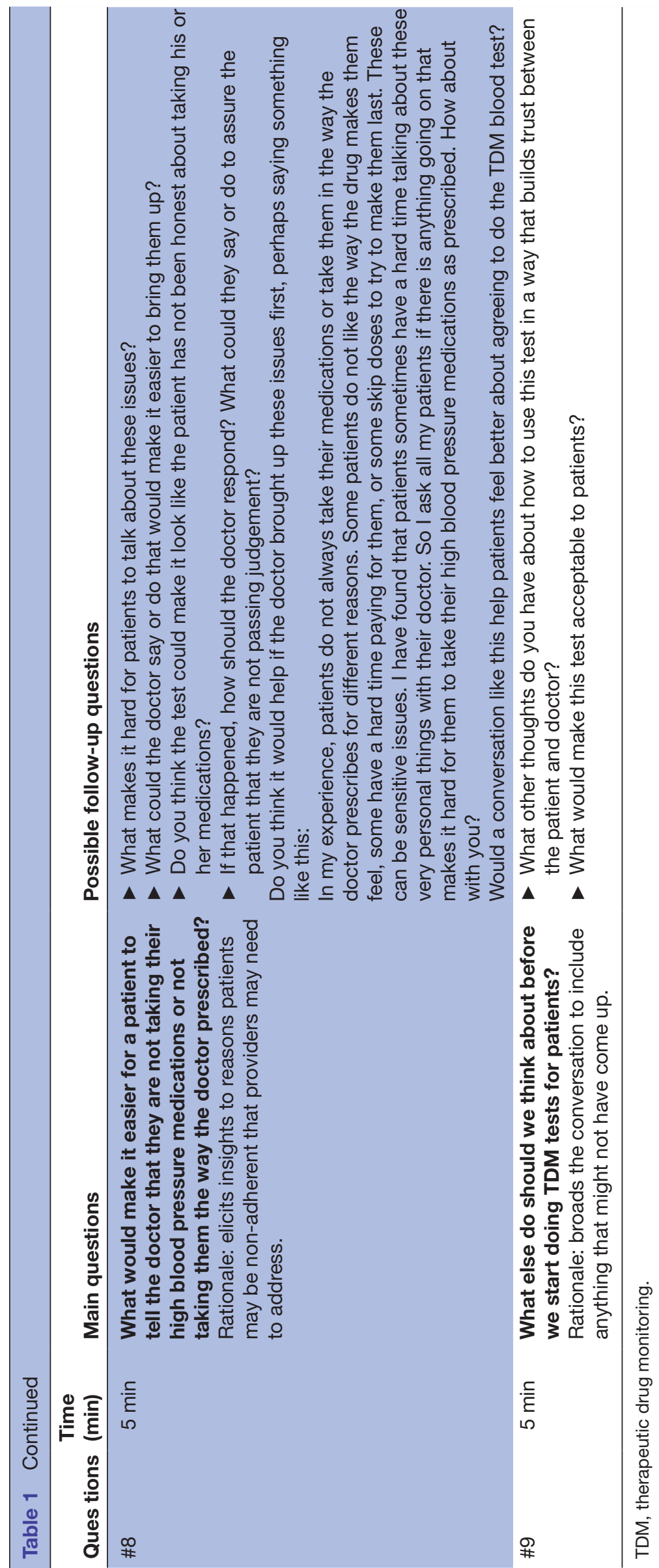


organisations serving minority, low-income populations in Dallas County. The meeting discussion was digitally recorded, transcribed and analysed in a similar fashion as the interview.

\section{Patient and public involvement}

The objective of this study was to glean patients' perspectives in the application of TDM in hypertension management. Patients were not directly involved in the production of the research study design. We supplemented patients' perspectives with input from the CAP programme, who are meant to represent and serve minority, low-income populations in Dallas County. Their perspectives also greatly contributed to our understanding of how to best use TDM. Input from the CAP programme was not used to implement or modify the study design.

\section{RESULTS}

During the course of 4 weeks in September 2018, 54 consecutive patients with uncontrolled hypertension ( $\mathrm{SBP} \geq 130 \mathrm{~mm} \mathrm{Hg}$ or DBP $\geq 80 \mathrm{~mm} \mathrm{Hg}$ despite treatment with $\geq 2$ antihypertensive drugs) who visited the General Internal Medicine or Cardiology clinic were identified from the electronic medical record. Among these subjects, 25 were excluded due to the inability to read or write English, 2 due to stage V CKD and 2 due to unwillingness to take their medications. Thirteen patients did not wish to participate and 11 were enrolled in the study. Patient participants included 8 women and 3 men ( 7 African Americans, 2 Hispanics and 2 non-Hispanic whites). The mean age of patients was $62 \pm 11$ years. The provider group consisted of 7 men and 3 women ( $80 \%$ non-Hispanic whites, $20 \%$ African Americans) with a mean age of $35 \pm 10$ years. The providers included: three internal medicine residents, three cardiology fellows, two physician assistants, one pharmacist and one cardiology attending physician). None of the providers were directly involved in the care of any of the patient participants.

\section{Patients' attitude and acceptability to TDM}

All 11 patients were aware of the potential impact of high $\mathrm{BP}$ and non-adherence to medications on health in terms of increasing the risk of stroke, heart disease, kidney disease and death. All patients identified themselves as adherers to medications as prescribed. When asked about the potential causes of medication non-adherence in general, five patients attributed it to inability to afford medications (which some patients felt uncomfortable mentioning to their providers), five to drug side effects, four to forgetfulness, two to being tired of having to take medications regularly and one to poor insight or health literacy. Overall $91 \%$ of patients (10 of 11) believed that TDM would be useful in their care and stated that they would agree to TDM testing. However, $63 \%$ (7 of 11) of patients expressed concerns that TDM could potentially negatively impact the physician-patient relationship. Two patients believed that the relationship could be strengthened and two others felt it would have no significant impact. When patients were asked which method of explanation TDM results would make them feel safe to admit their suboptimal adherence, $54 \%$ patients believed that they would feel at ease if providers acknowledged medication non-adherence and the underlying reasons, such as cost, inconvenience or forgetfulness, as a common problem. Honest communication by the providers was considered a major factor in facilitating admission of non-adherence in $45 \%$ patients. Empathy is thought be a major factor in $27 \%$ of patients. Providing education and communication to the patients regarding the adverse health consequences of the non-adherent behaviour is felt to be a major factor that triggers patient's admittance to non-adherence in $27 \%$. Other potentially positive aspects of TDM mentioned by the patients included enhancing provider's commitment to help patients (18\%), trust $(9 \%)$, truthfulness $(18 \%)$ and warm or nurturing personality of providers $(9 \%)$. The theme and codes of patients' views are shown in table 2.

\section{Providers' attitude and acceptability to TDM}

Similar to the patients' views, providers $(n=10)$ believed that inability to afford medication, forgetfulness, poor self-efficacy, concerns about medication side effects, pill burden and poor health literacy were major factors contributing to non-adherence. All providers thought TDM was a good idea and should be used regularly. Forty per cent of clinicians believed that TDM could strengthen the patient-provider relationship, particularly in patients in whom non-adherence was initially suspected but TDM demonstrated evidence of adherent behaviour. The same group of providers, however, believe that the results may have a negative effect in others for whom TDM confirmed medication nonadherence. Three providers $(30 \%)$ believed that TDM results would have no impact on the physician-patient relationship. Two providers $(20 \%)$ believed that TDM would negatively impact the patient-provider relationship. All providers felt TDM was potentially useful in identifying and solving non-adherence to antihypertensive drugs, if the counselling was delivered in a sensitive manner. When providers were asked how best to explain TDM results, half believed that acknowledging common obstacles to adherence (forgetfulness, side effects, costs and so on) and offering assistance accordingly would make the patients more comfortable revealing non-adherence. Other ideas about discussing TDM results and non-adherence included: building trust and rapport $(50 \%)$, using non-judgmental language $(40 \%)$, emphasising honesty (20\%) and using it as an opportunity to increase health literacy $(20 \%)$. The theme and codes of providers' views are shown in table 3.

\section{Input from the PCOR Center CAP}

The results of the patient and provider interviews were subsequently shared with our PCOR Center CAP. 


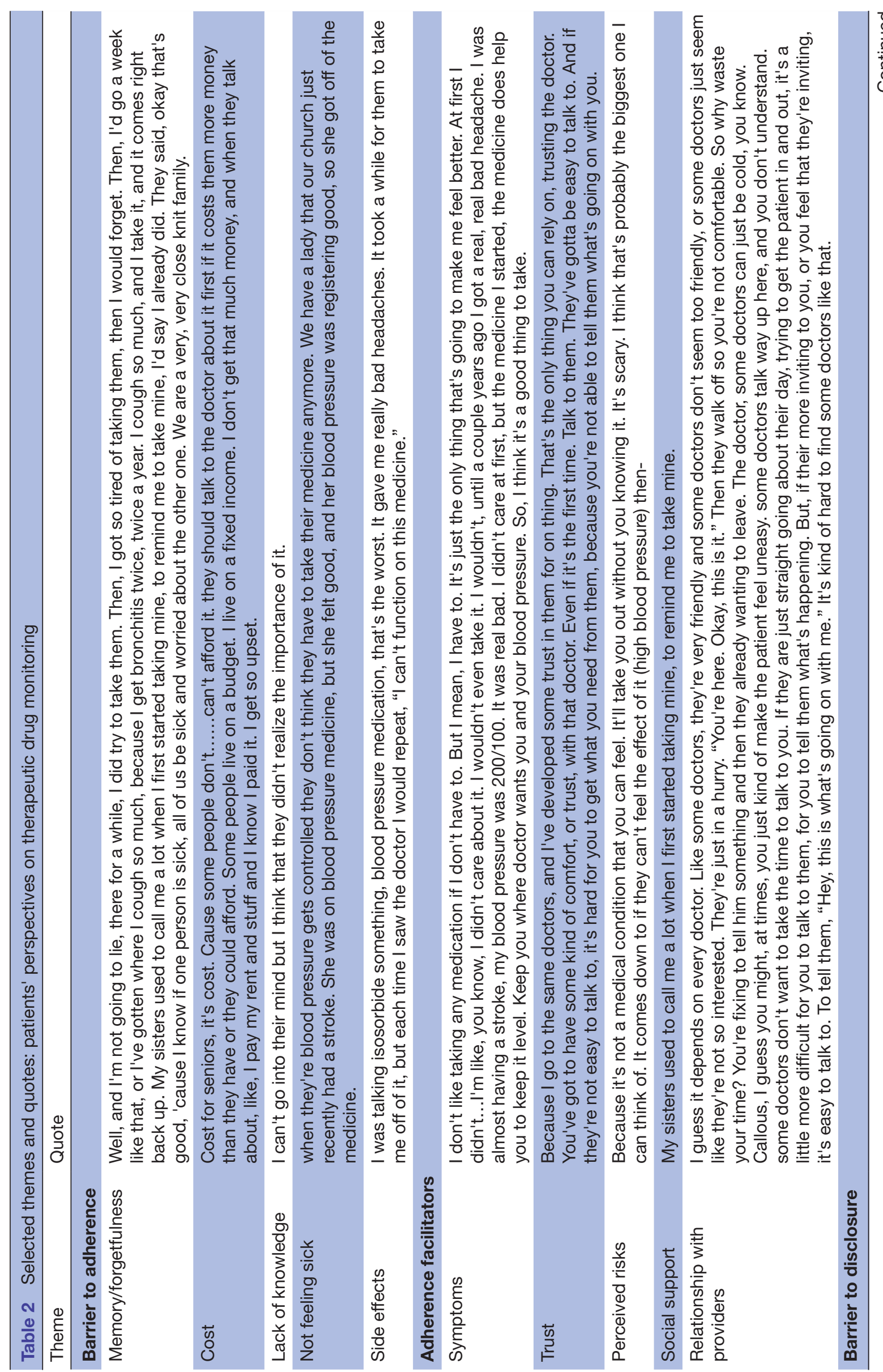




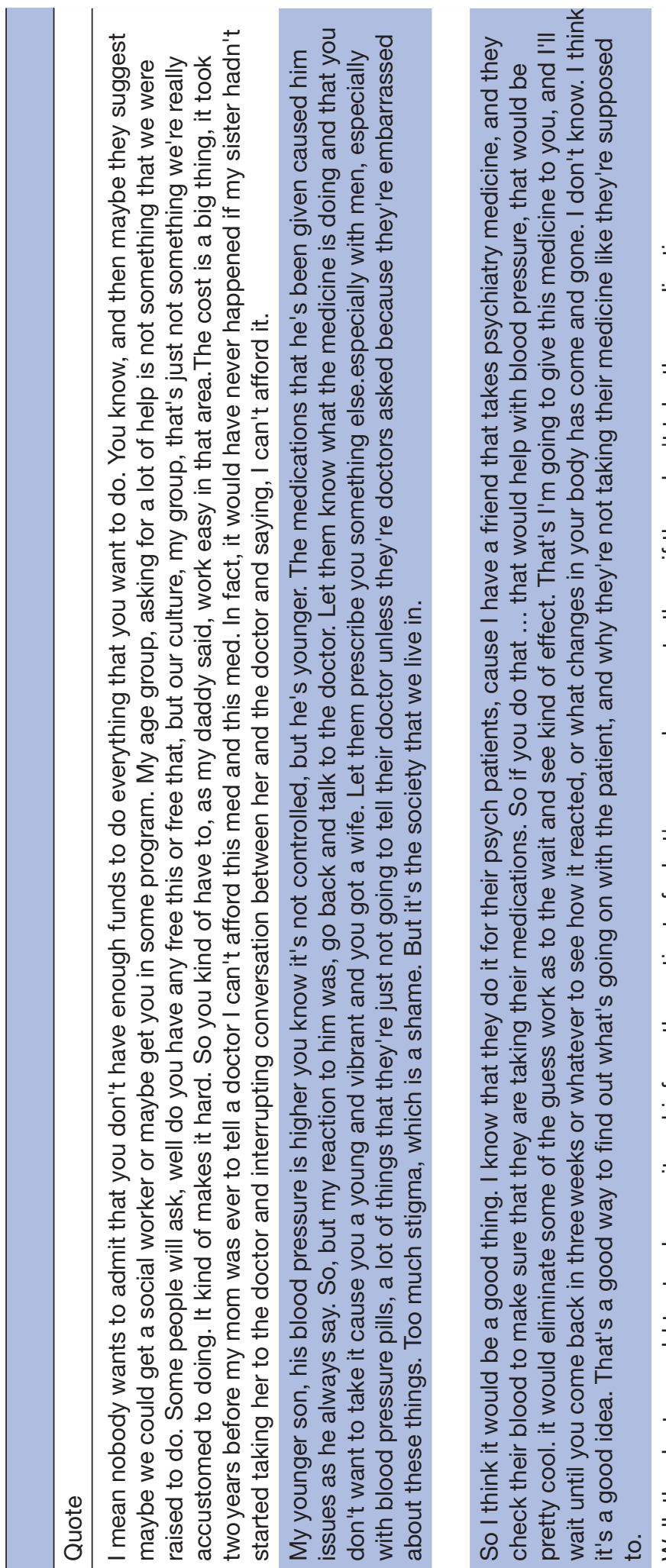

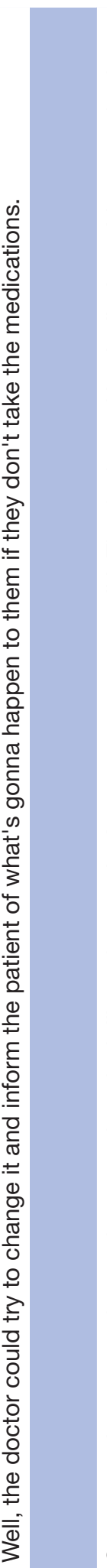

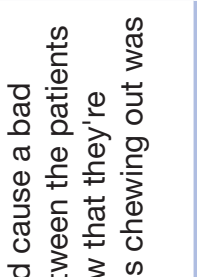

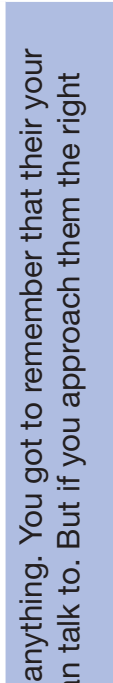

응

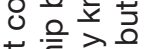

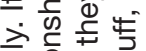
元.을

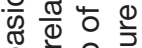
응 क రृ巳 क्ष

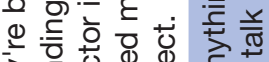
बे

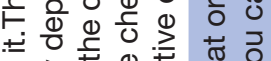
元 일

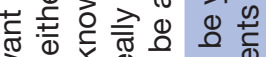
3 응은

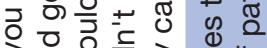

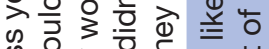

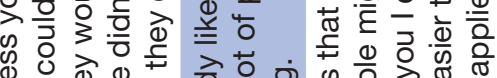

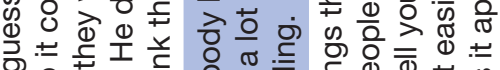
б 0 . の 음 $\overline{0}$ टँक कि

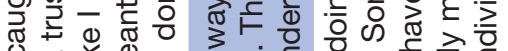

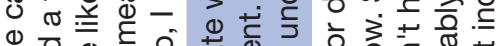
® 을 ⿺辶寸

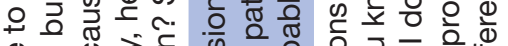

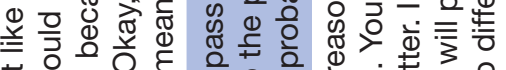
\pm 일 ह

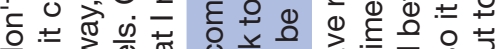

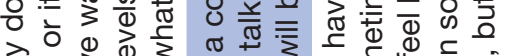
बे

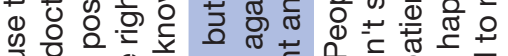

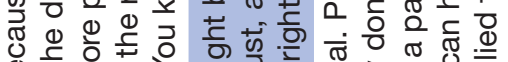

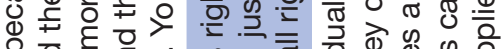

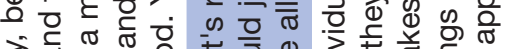

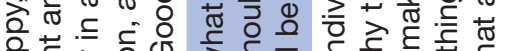

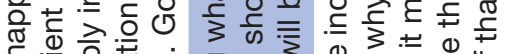
टत्ञ

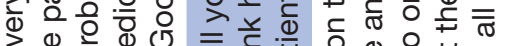

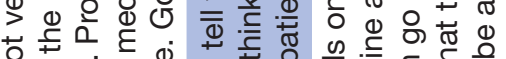
다에

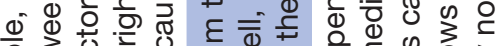
员

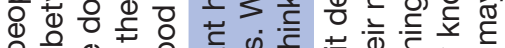

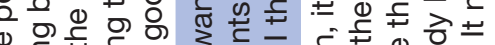

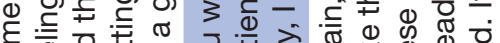
ウ 


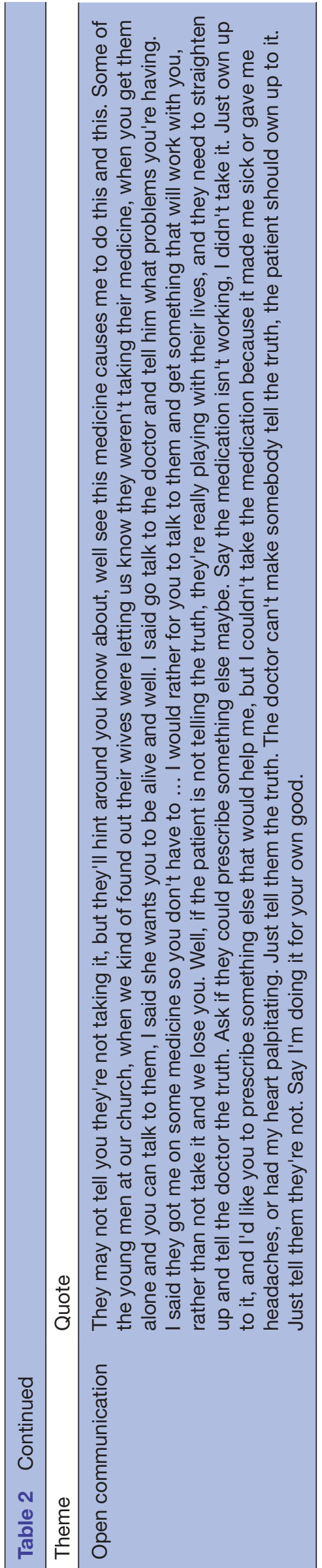

Thirteen CAP members were present in the meeting with the study investigators (RC, KBS, BE and WV). The CAP members acknowledged that some patients might feel embarrassed or angry by test results that are discordant from their self-reported adherence behaviour and that some patients have difficulty admitting financial barriers to medication adherence. One CAP member believed that provider should not 'sugar coat' the results and tell the patient upfront of the non-adherent behaviour. Most CAP members, however, believed that by acknowledging medication non-adherence as a common problem that most patients had to struggle to overcome obstacles would normalise the challenge and increase the likelihood of a productive discussion of their own nonadherence with providers. At the end of the discussion, all of the CAP members agreed that TDM was potentially useful and suggested several different ways to present the drug testing results to patients that would be acceptable, respectful, not damage patient-provider trust, and be more conducive to problem solving rather than placing blame.

\section{DISCUSSION}

Our study explored positive and negative attitudes towards TDM among low-income patients and providers who treat this population to better understand potential barriers and facilitators to using TDM in real-world practice.The major findings from the study are threefold. First, most patients with hypertension and their providers considered TDM a useful tool in monitoring adherence to medications. Second, more than half of patients and providers initially expressed concerns about the potential negative impact on the relationship if the results of TDM suggested non-adherent behaviour. Third, most patients believed that presenting TDM results in a collaborative, non-judgmental manner and exploring reasons underlying suboptimal adherence directly with the patient may lead to improved adherence and health outcomes. Remarkably, providers shared a similar view to the patients when discussing TDM results.

Adherence to medications can be monitored by several methods, including patient self-report, detailed questionnaires, pill counts, prescription fill rates, direct observation, electronic pillboxes or ingestible digital pills. ${ }^{13-15}$ Although self-report is often used to assess adherence, patients tend to overestimate their adherence to antihypertensive medications when compared with electronic pillbox data. ${ }^{15}$ Physicians' ability to predict patients' adherence to antihypertensive medications in the primary care setting is notoriously poor. ${ }^{16}$ Prescription fill rates may not be accurate if patients receive automatic prescription refills but do not ingest the dispensed medication. ${ }^{17}$ On the other hand, more accurate techniques, such as electronic monitoring or digital pill technology, are limited by the high cost which is not covered by any insurance carrier in the USA. ${ }^{2}$ In contrast to electronic monitoring, TDM is covered by insurance carriers in the USA and 


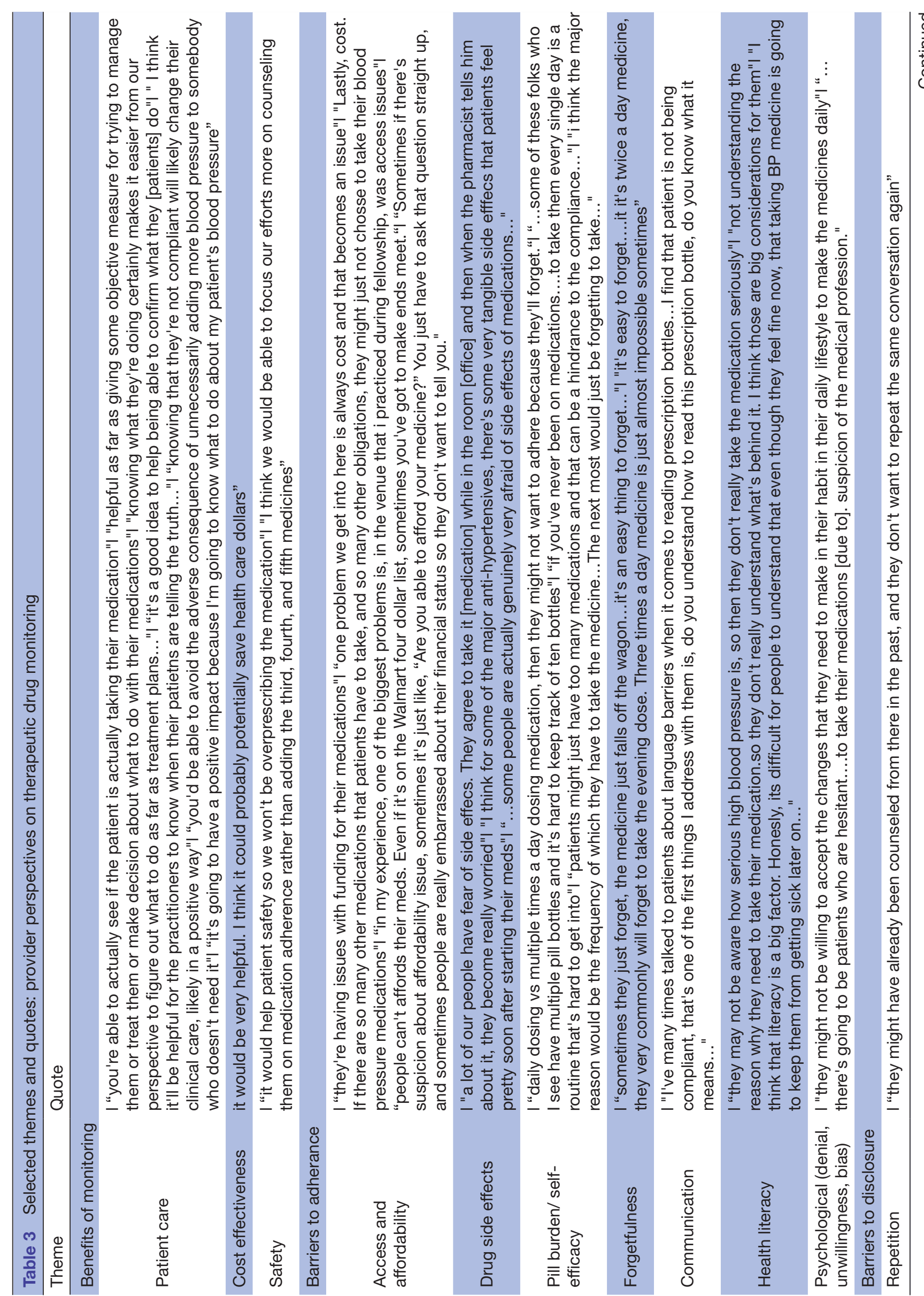




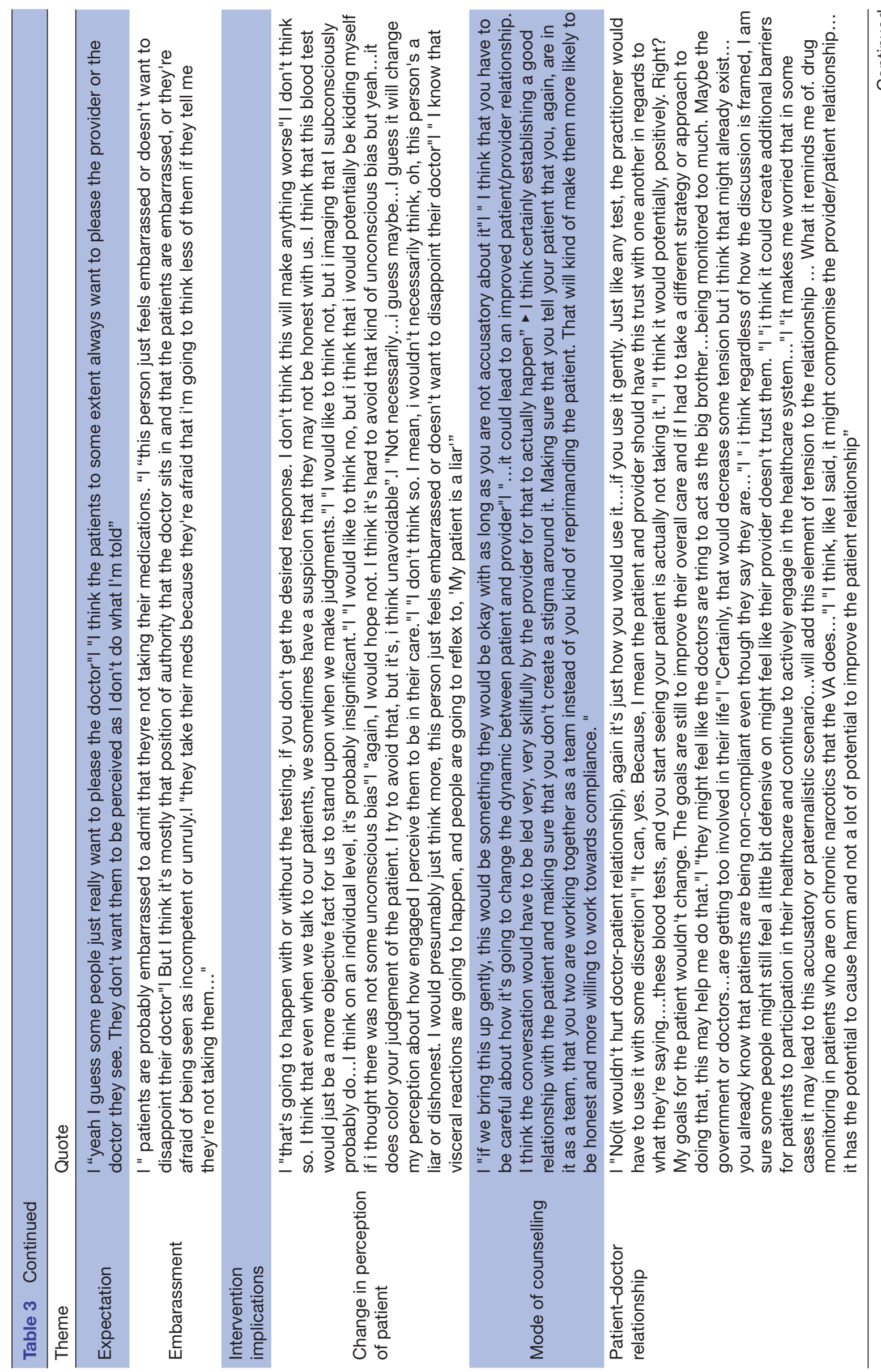




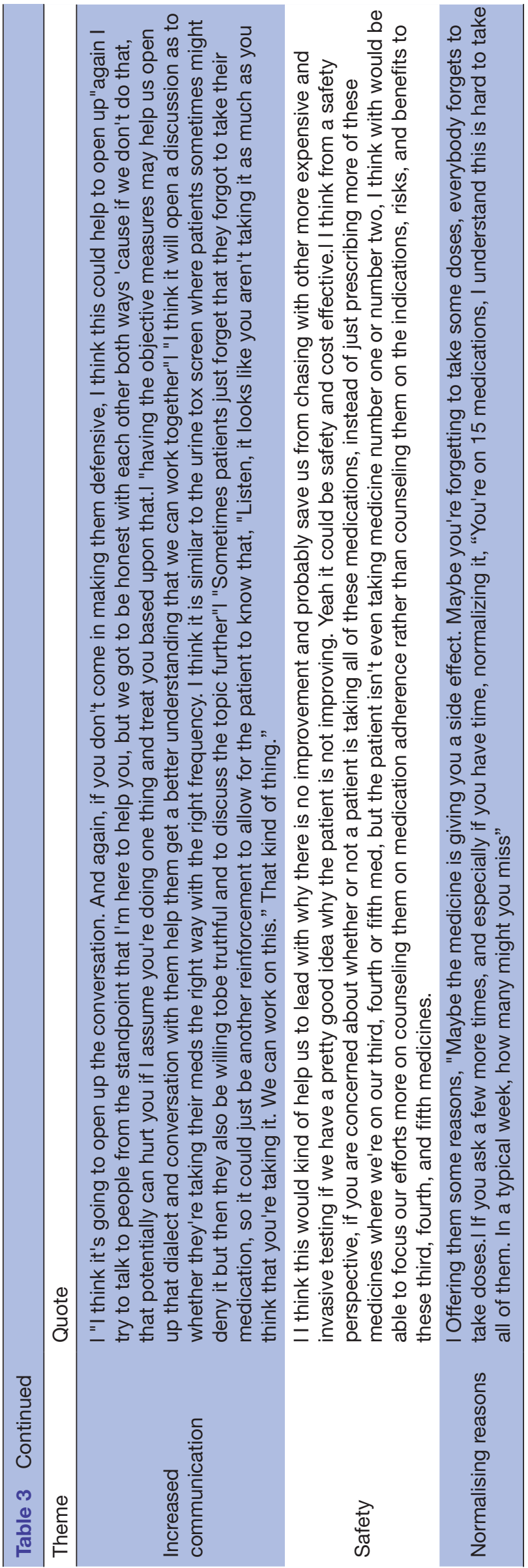

many countries worldwide. ${ }^{2}$ To our knowledge, our study represents the first that explores patients' and providers' attitudes in adopting TDM in routine practice.

We were encouraged that patients and provider identified similar reasons for medication non-adherence, barriers to disclosing them to providers, as well as the potential benefit of TDM to both hypertension control and the patient-provider relationship. As expected, both patients and providers expressed concerns about the potential impact of TDM on trust. However, patients and providers believed the benefits of an honest discussion about adherence outweighed the potential risks to the patient-provider relationship. Specifically, patients recommended that providers acknowledge that medication non-adherence is a common but addressable challenge. More importantly, many patients recommended providers ask if they experienced specific barriers to adherence such as drug cost or forgetfulness as patients reported often having difficulty volunteering information upfront due to shame and guilt. Patients also suggested that providers discuss medication nonadherence in the context of how hard it can be for many people to change all kinds of health behaviours like diet and physical activity. This approach would both decrease stigma and shame about this common occurrence, and set the stage for an honest, patientcentred discussion for practical strategies for improving adherence and hypertension control. In our interviews, providers who raised concerns about TDM adversely impacting patient trust also indicated that framing and presentation of adherence mattered and could mediate their concern. Overall, this suggests that provider education and training will be important if TDM is to be incorporated into routine clinical practice.

Presenting findings to the CAP provided the added advantage of providing a collective and peer-based approach to 'member-checking,' a qualitative technique where researchers test their understanding or interpretation of findings with participants. In our study, we found CAP reactions to the issues of non-adherence were remarkably similar to those patients we interviewed, validating our findings. Community members recognised adherence as a challenge to patients. Their remarks emphasised their shared experience as patients with first-person accounts of their experience. Based on input from patients, providers and CAP members, we propose several key factors that are essential in enhancing patient-provider communication of TDM results and obstacles to medication adherence as shown in figure 1. Future work to design interventions might benefit from more explicit engagement with family members and other caregivers to identify their role in managing patient adherence that might complement TDM efforts in the clinic.

This was a qualitative methods pilot study so our small sample size is a potential limitation. The study results may not be applicable to many patients who were excluded from the study due to inability to read or write English or 
Factors Enhancing Patient-Provider Communication of TDM Results and Obstacles to Medication Adherence

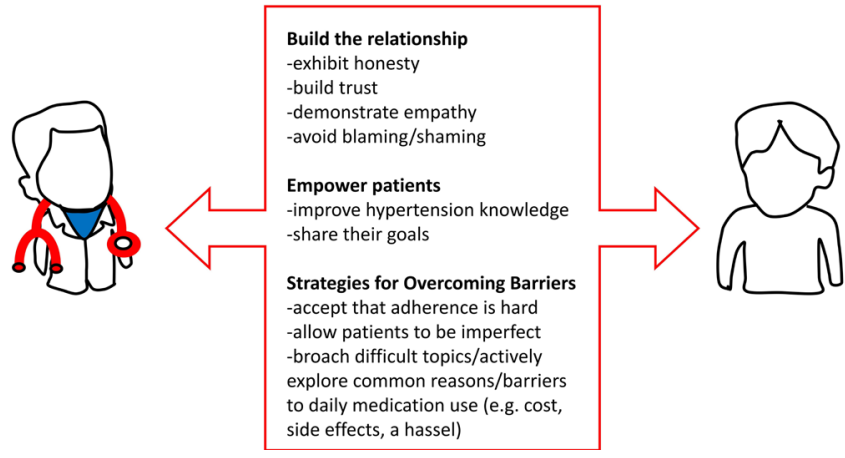

Figure 1 Proposed factors enhancing patient-provider communication of therapeutic drug monitoring (TDM) results and obstacles to medication adherence.

verbally expressed non-adherence to medication. Furthermore, more than half of eligible patients refused to participate and their views regarding TDM may differ from those participating in our study. During the interview, we did not address the limitation of TDM in the capturing drug levels with short half-life or long sample collection time since last ingestion which may lead to misinterpretation of results by the clinicians. Nevertheless, we were able to explore attitudes in depth among the patients enrolled in the study, and the themes that emerged are concordant with the literature on medication non-adherence. Additionally, participants were patients who were predominantly non-White, low income, uninsured individuals in an urban safety health system, and clinicians who care for these vulnerable populations so generalisability to other settings is uncertain. On the other hand, such patients and settings are most heavily impacted by non-adherence and poor hypertension control.

In summary, the idea of TDM was well-accepted by patients and providers. Despite the fact that both groups expressed concerns that TDM could potentially negatively impact the physician-patient relationship, more than $90 \%$ of patients and providers believed that TDM constituted an effective tool in identifying and overcoming barriers to adherence, especially if providers could address this common and important problem in a respectful, patient-centred fashion.

\section{Author affiliations}

${ }^{1}$ Internal Medicine, University of Texas Southwestern Medical Center at Dallas, Dallas, Texas, USA

${ }^{2}$ Hypertension Section, Internal Medicine, University of Texas Southwestern Medical Center, Dallas, Texas, United States

${ }^{3}$ General Internal Medicine Division, Internal Medicine, The University of Texas Southwestern Medical Center, Dallas, Texas, USA

${ }^{4}$ Department of Population and Data Sciences, University of Texas Southwestern Medical Center, Dallas, Texas, United States

${ }^{5}$ Cardiology Division, Internal Medicine, University of Texas Southwestern Medical Center, Dallas, United States

${ }^{6}$ Department of Applied Clinical Research, University of Texas Southwestern Medical Center, Dallas, Texas, United States

${ }^{7}$ Department of Psychiatry, The University of Texas Southwestern Medical Center, Dallas, Texas, USA
${ }^{8}$ Center for Innovation and Value, Parkland Health and Hospital System, Dallas, Texas, USA

Twitter Hamza Lodhi @realHamzaLodhi, Sandeep R Das @sandeepdasmd and Wanpen Vongpatanasin @DrWanpen

Contributors WV is the senior author. KBS and SS interviewed participants. KBS, $\mathrm{HL}, \mathrm{BW}, \mathrm{SAS}$ and SS organised recorded transcripts. KBS, RC and WV coded the transcripts. WV conceived the study. BE, EAH, SJCL and WV contributed to study design. WV, RC, BE, SJCL, SAS, RBJ, SD, EAH and KBS contributed to the final manuscript. WV and EAH secured funding support. WV is the guarantor.

Funding This project was supported by grant number R24HS022418 from the Agency for Healthcare Research and Quality (to EAM, BE, SCL, and WV), the UT Southwestern T32 training grant in Nephrology (to RC), and the UT Southwestern O'Brien Kidney Center and the Pak Center of Mineral Metabolism and Clinical Research (to WV).

Competing interests None declared.

Patient and public involvement Patients and/or the public were not involved in the design, or conduct, or reporting, or dissemination plans of this research.

Patient consent for publication Not required.

Ethics approval This study was approved by the University of Texas Southwestern Medical Center Institutional Review Board.

Provenance and peer review Not commissioned; externally peer reviewed.

Data availability statement Data are available upon reasonable request. All data acquired from this prospective analysis will be shared with the public scientific community upon request. Requests can be made to wanpen.vongpatanasin@ utsouthwestern.edu. This includes original recorded transcripts and coded data. Data will be made available upon publication with no end date. The lead author (KBS) affirms that the manuscript is an honest, accurate and transparent account of the study being reported; that no important aspects of the study have been omitted; and that any discrepancies from the study as planned have been explained.

Open access This is an open access article distributed in accordance with the Creative Commons Attribution Non Commercial (CC BY-NC 4.0) license, which permits others to distribute, remix, adapt, build upon this work non-commercially, and license their derivative works on different terms, provided the original work is properly cited, appropriate credit is given, any changes made indicated, and the use is non-commercial. See: http://creativecommons.org/licenses/by-nc/4.0/.

ORCID iD

Kevin B Schesing http://orcid.org/0000-0003-1488-6482

\section{REFERENCES}

1 Chowdhury R, Khan H, Heydon E, et al. Adherence to cardiovascular therapy: a meta-analysis of prevalence and clinical consequences. Eur Heart J 2013;34:2940-8.

2 Brinker S, Pandey A, Ayers C, et al. Therapeutic drug monitoring facilitates blood pressure control in resistant hypertension. J Am Coll Cardiol 2014;63:834-5.

3 Pandey A, Raza F, Velasco A, et al. Comparison of Morisky medication adherence scale with therapeutic drug monitoring in apparent treatment-resistant hypertension. J Am Soc Hypertens 2015;9:420-6.

4 Velasco A, Chung O, Raza F, et al. Cost-Effectiveness of therapeutic drug monitoring in diagnosing primary aldosteronism in patients with resistant hypertension. J Clin Hypertens 2015;17:713-9.

5 Vrijens B, Vincze G, Kristanto P, et al. Adherence to prescribed antihypertensive drug treatments: longitudinal study of electronically compiled dosing histories. BMJ 2008;336:1114-7.

6 Chang TE, Ritchey MD, Park S, et al. National rates of nonadherence to antihypertensive medications among insured adults with hypertension, 2015. Hypertension 2019;74:1324-32.

7 Gupta P, Patel P, Štrauch B, et al. Biochemical screening for nonadherence is associated with blood pressure reduction and improvement in adherence. Hypertension 2017;70:1042-8.

8 Chung O, Vongpatanasin W, Bonaventura K, et al. Potential costeffectiveness of therapeutic drug monitoring in patients with resistant hypertension. $J$ Hypertens 2014;32:2411-21.

9 Armstrong K, Ravenell KL, McMurphy S, et al. Racial/ethnic differences in physician distrust in the United States. Am J Public Health 2007;97:1283-9. 
10 Committee. P-C. The PCORI methodology report; 2019.

11 Creswell JW, Miller DL. Determining validity in qualitative inquiry. Theory Pract 2000;39:124-30.

12 Miles MB, Huberman AM. Qualitative data analysis: an expanded Sourcebook. 2nd ed. Thousand Oaks, CA: Sage Publications, 1994.

13 Burnier M, Egan BM, Egan Brent M. Adherence in hypertension. Circ Res 2019;124:1124-40.

14 Feyz L, Bahmany S, Daemen J, et al. Therapeutic drug monitoring to assess drug adherence in assumed resistant hypertension: a comparison with directly observed therapy in 3 nonadherent patients. J Cardiovasc Pharmacol 2018;72:117-20.
15 Zeller A, Ramseier E, Teagtmeyer A, et al. Patients' self-reported adherence to cardiovascular medication using electronic monitors as comparators. Hypertens Res 2008;31:2037-43.

16 Zeller A, Taegtmeyer A, Martina B, et al. Physicians' ability to predict patients' adherence to antihypertensive medication in primary care. Hypertens Res 2008;31:1765-71.

17 Ruzicka M, Leenen FHH, Ramsay T, et al. Use of directly observed therapy to assess treatment adherence in patients with apparent treatment-resistant hypertension. JAMA Intern Med 2019.

doi:10.1001/jamainternmed.2019.1455. [Epub ahead of print: 17 Jun 2019]. 